\section{Christoph Bachmann}

\section{Auf verschiedenen Ebenen}

$\mathrm{Ob}$ in einer Apotheke eine Teemischung oder eine einzelne Arzneidroge verkauft wird, ob ein Küchenchef in einem Restaurant ein Menü mit auserlesenen Gewürzen verfeinert, ob in einem Wellness-Center jemand eine wohltuende Kräuterpackung auf sich wirken lässt oder ob in einer pharmazeutischen Firma ein pflanzliches Arzneimittel hergestellt wird: Immer kann das Familienunternehmen Dixa AG (Kasten 1) aus St. Gallen daran beteiligt sein. Denn die St. Galler Firma verarbeitet als grösste Schweizer Kräuterfirma jährlich über 1000 Tonnen Kräuter und beliefert damit verschiedenste Anwender von Kräutern. Die Rohstoffe werden weltweit eingekauft und am Firmensitz in St. Gallen verarbeitet.

\section{Über 700 Arten}

Mit über 700 Arten an Arzneipflanzen sowie Kräutern und Gewürzen für Lebensmittel und Kosmetik verfügt das Familienunternehmen über dasgrösste Sortiment der Schweiz und ist Lieferant für die unterschiedlichsten Branchen:

- Pharma- und Heilmittelindustrie;

- Lebensmittelindustrie;

- Kosmetik- und Nahrungsergänzungsmittelindustrie;

- Spezialitäten- und Detailhandel;

- Fachhandel des Gesundheitswesens;

- Veterinär- und Spezialfuttermittelindustrie.

In Kreisen von Apotheken und Drogerien ist die Dixa in erster Linie als Lieferant von Heilkräutern bekannt. Und als solche spielt die

\title{
Dixa AG: Das Familienunternehmen mit Tradition
}

Wenn man über die komplementärmedizinischen Firmen der Schweiz spricht, denkt man zuerst an die Hersteller von Fertigpräparaten. Sie produzieren oder vertreiben die Präparate, welche die Mehrheit der in Apotheken und Arztpraxen eingesetzten ausmachen. Weniger im Rampenlicht stehen Firmen, die Kräuter verarbeiten und Tinkturen herstellen oder als Lieferant von veredelten Rohstoffen agieren. Die Firma Dixa AG in St. Gallen ist seit beinahe 100 Jahren auf diesem Gebiet aktiv.

Firma auch eine wichtige Rolle im Schweizer Gesundheitswesen und in der Schweizer Komplementärmedizin. Das St. Galler Unternehmen macht rund die Hälfte seines jährlichen Umsatzes mit Anbietern des Gesundheitswesens, die andere Hälfte mit Firmen der Lebensmittel-, Kosmetik- und Futtermittelindustrie.

Da die Nachfrage nach biologisch angebauten Rohstoffen, sprich biologischen Arzneipflanzen, immer grösser wird, hat die Dixa AG ein gutes Netzwerk von Anbauern in der Schweiz und im Ausland aufgebaut. Dies gewährleistet, dass die als biologisch bezeichneten Kräuter auch wirklich diesem Standard entsprechen. Die im Jahr 2005 erfolgte Übernahme der Hans Kennel AG in Baar bedeutete einen wichtigen Schritt in diesen Bemühungen. Denn diese innerschweizer Firma ist ein ausgesprochener Spezialist für Bio-Qualität und Kräuter aus Schweizer Anbau. «Leider ist es uns aber oft nicht möglich, unsere nach der europäischen Pharmakopoe untersuchten Heilpflanzen in biologischer Qualität anzubieten. Das Angebot bzw. die Auswahl ist häufig zu klein, und selbst wenn Ware vorhanden ist, heisst das noch nicht, dass sie den hohen Ansprüchen der PHEUR entspricht. Viele unserer Produkte stammen aber sowieso aus Wildsammlung, sind also auch biolo- gisch, einfach nicht zertifiziert», sagt Bettina Wild.

\section{Qualität und Nachhaltigkeit}

«Qualität und Nachhaltigkeit sind für uns zwei äusserst wichtige Kriterien beim Einkauf der Kräuter», erklärt Stefan Postl, der zusammen mit Bettina Wild seit 2010 das Familienunternehmen leitet (Kasten 2). «Manchmal erhalten wir verlockende Offerten. Wenn wir aber sehen, dass die angebotenen Kräuter nicht der Qualität entsprechen, der wir uns verpflichtet haben, dann gehen wir nicht darauf ein.» Bettina Wild ergänzt: «Und um dies zu gewährleisten, besuchen wir unsere Lieferanten weltweit und regelmässig.»

\section{Vielfältige Produktion}

Wer sich einen Kräuterlieferant einfach als eine Firma vorstellt, bei der nur die eingekauften Kräuter in grossen Lagerhallen gelagert und weiterverkauft werden, liegt bei der Dixa AG falsch, denn im aus dem Jahr 1898 stammenden Firmengebäude (Abb. 1) werden fast alle Rohstoffe auf den unterschiedlichsten Maschinen verarbeitet und veredelt. So befinden sich in St. Gallen verschiedene Schneideanlagen und Mühlen, die für jede

\section{KARGER}

Fax +49761 4520714 Information@Karger.com www.karger.com (c) 2014 S. Karger GmbH, Freiburg 
1916 - Gründung der Firma Willi Dix, Dixa-Werke, Rorschach

1931 - Übernahme der Geschäfte seines Onkels durch David Eduard Wild und Gründung der Aktiengesellschaft Dixa AG, St. Gallen

1933 - Gründung der Schwesterfirma Extracta AG, St. Gallen. Zuerst in der Produktion von Flüssigextrakten tätig, konzentriert sich das Unternehmen später auf die Lohnherstellung von Tabletten und Dragees

1956 - Die Lagerkapazitäten werden mit einem Erweiterungsbau vergrössert

1985 - Die Söhne Reto und Jürg Wild übernehmen die Geschäftsleitung

1989 - Nach zweijähriger Planungs- und Bauzeit kann der Erweiterungsbau West bezogen werden

1994 - Zertifizierung nach ISO 9001

1997 - Integration der Extracta AG in die Dixa AG, Austritt von Jürg Wild aus der Geschäftsleitung

2004 - Zertifizierung nach BRC Global Standard Food

2005 - Übernahme der Hans Kennel AG, Baar

2009 - Unerwarteter Tod des Inhabers und Geschäftsführers Reto Wild

2010 - Eintritt von Bettina Wild und Stefan Postl, der vierten Generation

Kasten 2. Fragen an Bettina Wild und Stefan Postl, Inhaber und Geschäftsführer der Dixa AG in vierter Generation

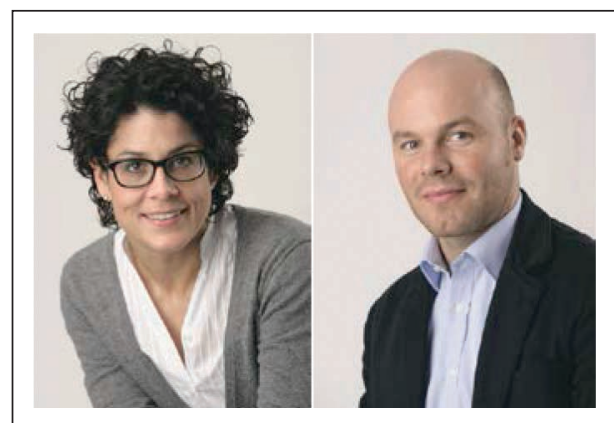

Frau Wild, Sie repräsentieren mit Herrn Stefan Postl zusammen die vierte Generation in Ihrem Familienunternehmen. Sind Sie also quasi mit Kräutern aufgewachsen?

Wild: Ja, wir hatten (und haben) zu Hause immer einen Krug frischen Kräutertee stehen, und mein Onkel hat mich als Kind bei Familienfeiern motiviert, frischen Pfeffer aus der Mühle über das Essen zu streuen. «Wir müssen die Gewürzhändler unterstützen», sagte er immer mit einem Zwinkern.

Heute verlagern immer mehr Firmen ihre Produktionsstandorte nach Osteuropa und Asien. Sie bleiben mit Ihren 60 Mitarbeitenden in St. Gallen. Wie schaffen Sie das?

Postl: Wir schaffen es, weiterhin in der Schweiz zu produzieren, weil wir eine Nische besetzen, viel Einsatzbereitschaft und Flexibilität zeigen und einen Namen für qualitativ hochwertige Heilkräuter und Gewürze haben.

Insbesondere das Wissen darum, wo und wie Heilkräuter in der richtigen Qualität beschafft oder angebaut werden, basiert auf jahrelanger Erfahrung. Unsere Naturprodukte können von Jahr zu Jahr ganz unterschiedliche Qualitäten aufweisen, z.B., weil es zu viel oder zu wenig geregnet hat oder weil es zu heiss oder zu kalt war. Auch eine unsorgfältige Ernte oder Trocknung kann die Qualität massiv beeinflussen. Wir arbeiten deshalb sehr eng mit unseren Anbauern und Sammlern zusammen und reinigen und verarbeiten in St. Gallen sämtliche Rohstoffe aus aller Herren Länder, sodass sie dem qualitätsbewussten Schweizer Kunden gefallen.

Die Dixa AG hat trotzdem mit Osteuropa zu tun, indem sie, wie auch andere Kräuterlieferanten, einen Grossteil der Kräuter aus diesen Ländern bezieht. Woher stammt diese Tradition?

Wild: In Osteuropa hat das Sammeln von Heilkräutern eine lange Tradition. Früher wurde das Know-how von Generation zu Generation weitergegeben, und es ist wohl so, dass diese Sammeltätigkeit nebst der Deckung des Eigenbedarfs auch als willkommener Zusatzverdienst betrachtet wurde.

Auch bei uns in der Schweiz wurde früher fleissig gesammelt. Mit den steigenden Einkommen wurde dieser Nebenerwerb aber immer weniger attraktiv. Hinzu kommt die - aufgrund intensiver Landwirtschaft - abnehmende Vielfalt der Heilpflanzen in unseren Breitengraden. Ganz im Gegensatz dazu verfügen viele osteuropäische Staaten noch über grosse, kaum berührte Landstriche.

\section{Wachsen diese Pflanzen in erster Linie in Kulturen oder als Wildwuchs?}

Postl: Viele der aus Osteuropa importierten Pflanzen stammen aus behördlich genehmigten und kontrollierten Wildsammlungen. Wir lassen jedoch auch einen Grossteil der «Standardkräuter» wie Melisse, Pfefferminze, Kamille und Baldrianwurzeln, um nur einige zu nennen, anbauen. 


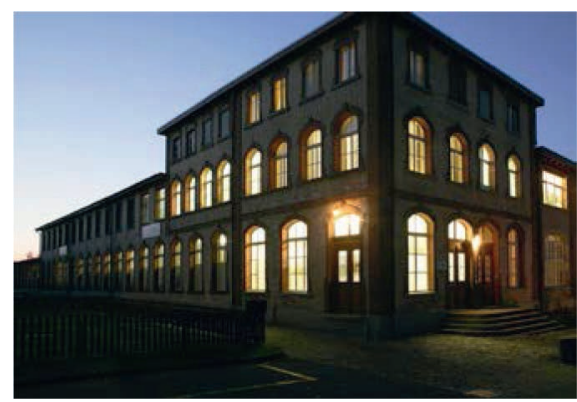

Abb. 1. Das aus dem Jahr 1898 stammende Firmengebäude der Dixa AG.

Arzneipflanze und für die unterschiedlichsten Qualitätsanforderungen genau die richtige Verarbeitung gewährleisten.

Da die Dixa AG auch sehr viele Kräutermischungen herstellt, findet sich in der Produktion eine beeindruckende Anzahl von Mischanlagen, mit denen geschnittene Kräuter, aber auch Pulver unterschiedlichster Mengen gemischt werden können.

Trotz aller Maschinen bleibt aber auch vieles sorgfältige Handarbeit. Markus Düring, Leiter Produktion, weist stolz darauf hin, dass grob geschnittene Kräuter, die z.B. für Teemischungen verwendet werden, mit einer Schaufel von Hand gemischt werden. So wird gewährleistet, dass möglichst wenige «sensible» Blätter und Blüten zu Bruch gehen.

\section{Qualitätskontrolle}

Damit Qualität nicht nur ein schönes Wort bleibt, sorgt ein Team von 9 Spezialisten und Spezialistinnen für die Qualitätssicherung und analytische Qualitätskontrolle im Labor. So wird sichergestellt, dass Qualität ein Begleiter aller Stufen ist, welche die Kräuter in der Firma durchlaufen, bis sie als Arzneidroge, Teemischung, Gewürz usw. an die Kundschaft geliefert werden. Die Qualitätskontrolle umfasst die Identitäts- und Gehaltsprüfung, aber auch die Rückstandanalytik (z.B. Prüfung auf den Einsatz der nicht erwünschten Pestizide). Dixa hat sich sowohl den Rahmenbedingungen und Regelwerken aus der Pharmaindustrie (GMP - Good Manufacturing Practice) als auch der Lebensmittelindustrie (ISO 9001:2008 und BRC Global Standard Food) verschrieben.

\section{Lohnherstellung}

Die Dixa AG kann auch auf eine lange Tradition in der Lohnherstellung für Apotheken und Drogerien zurückblicken und besitzt eine Liste von mehr als 3000 individuellen Rezepten für verschiedenste Anwendungen. Apotheken und Drogerien können sich zum Thema Hausspezialitäten von der Dixa beraten und diese herstellen lassen. Bei Teemischungen ist dies ab Mengen von $5 \mathrm{~kg}$ möglich, bei Tabletten ab 100000 Stück, auf Verlangen auch fertig konfektioniert.

\section{Teufelskralle}

Seit Jahren vertreibt die Dixa AG Teufelskrallenwurzel-Tabletten, die seit 2010 in der Schweiz auch als Fertigarzneimittel Liste D registriert sind. Dabei handelt es sich um Tabletten mit $182 \mathrm{mg}$ TeufelskrallenwurzelPulver (Harpagophyti radicis pulvis) pro Tablette, standardisiert auf $2 \mathrm{mg}$ Harpagoside. Im Unterschied zu anderen Produkten auf dem Markt wird für diese Tablette nicht ein standardisierter Extrakt, sondern die gesamte vermahlene Wurzel verwendet. Die aus dem südlichen Afrika stammende Teufelskralle (Harpagophytum procumbens) wird in den westlichen Ländern seit etwa 10 Jahren intensiv erforscht und weist eine klinisch dokumentierte Wirkung bei degenerativen Gelenkerkrankungen auf. Weitere Arzneimittel unter eigenem Namen auf den Markt zu bringen, ist nicht geplant. «Wir fokussieren uns auf die qualitativ hochwertige Herstellung und Dokumentation von Hausspezialitäten oder bereits registrierten Arzneimitteln. Wir bedauern sehr, dass der bürokratische und finanzielle
Aufwand zur Registrierung von Phytoarzneimitteln kontinuierlich ansteigt und damit vielen althergebrachten Rezepturen den Todesstoss versetzt», sagt Bettina Wild.

\section{Gewürzmischungen}

Ein anderes wichtiges Standbein der Firma Dixa AG sind Gewürzmischungen, die im Hause entwickelt und an viele Abnehmer verschickt werden. Darunter sind Grossverteiler, viele bekannte Markenartikelhersteller der Lebensmittelindustrie, aber auch Restaurants und Hotels. Ob man eine authentische Curry-Mischung bestellen will oder eine Gewürzmischung mit einem neuen Geschmack sucht, beim St. Galler Unternehmen kann man fündig werden. Denn die firmeninternen Spezialisten entwickeln immer wieder neue Mischungen und Geschmacksrichtungen. Hier ein Auszug aus der Gewürzliste der Firma:

- Dixa Café de Paris

- Dixa Chili Mischung mild

- Dixa Grillgewürz

- Dixa Gurkengewürz

- Dixa Herbes de Provence

- Dixa Italienische Kräutermischung

\section{Wellness}

Da Kräuter im stark wachsenden Wellness-Markt eine wichtige Rolle spielen, ist das St. Galler Familienunternehmen natürlich auch in diesem Segment tätig und bietet eigene Mischungen an oder stellt von Kunden gelieferte Rezepte her. Das Sortiment der Wellness-Produkte umfasst Kräutertees zur Entspannung, Kräuter- und Blütenbäder sowie Kräuter für Kräuterstempelmassagen. "Auch in diesem Bereich sind wir sehr aktiv», sagt Bettina Wild bestimmt, «den Trend zurück zur Natur, zum Natürlichen und Authentischen beobachten wir mit Interesse. Wir finden es sehr wichtig, 
dass die im Wellness-Bereich eingesetzten Kräuter und Kräutermischungen von höchster Qualität sind. Denn nur so wird die angestrebte ganzheitliche Entspannung und Erholung erreicht!»

Bei den Kräuterbädern findet man auf der Sortimentsliste z.B. ein Dixa Kräuterbad Aphrodixa, das ein feines Wortspiel darstellt: Aphrodixa ist eine Verbindung des Firmennamens Dixa mit der griechischen Liebesgöttin Aphrodite.

\section{Ausblick}

In welche Richtung steuert das Familienunternehmen in der nahen Zukunft? Wird das eine oder andere Standbein an Bedeutung gewinnen? Welche Märkte sind die schwierigsten, welche die erfolgversprechendsten? «Wir wollen weiterhin die Spezialistin für die Verarbeitung und Veredelung von Heilkräutern und Gewürzen in der Schweiz sein und diesen Markt auch ausbauen. Wir wis- sen, dass wir den Produktionsstandort Schweiz nur halten können, wenn wir einwandfreie Qualität liefern, flexibel sind und unsere Kunden gut bedienen. Die Märkte für Lebensmittel wie auch pflanzliche Arzneimittel sind unter Druck, aber wir betrachten dies als Chance, unser Unternehmen fit zu halten und den 〈grossen Playern〉 zwischendurch ein Bein zu stellen», sagt Stefan Postl. 